\title{
HTML 5 Support for an Accessible User-Video-Interaction on the Web
}

\author{
Lourdes Moreno, Paloma Martínez, Ana Iglesias, and María Gonzalez \\ LaBDA Group, Computer Science Department, Universidad Carlos III de Madrid \\ \{lmoreno,pmf, aiglesia,mgonzal\}@inf.uc3m.es
}

\begin{abstract}
Multimedia content covers the Web, and we should provide access to all people. For this reason, it is very important to take into account accessibility requirements in the player to avoid barriers and to ensure access to this multimedia content as well as their resources. One of the most frequent barriers is the technological obstacle: the necessity for the user to install the required plug-ins in to order to access video. The new standard HTML5 provides a solution to this problem. However, it does not fully support accessibility requirements of $\mathrm{W} 3 \mathrm{C}$ standards, including $\mathrm{WCAG}$ and interaction requirement of UAAG. This paper ${ }^{1}$ introduces an overall study of this new standard in relation to accessibility requirements for the players as well as an accessible HTML5 Media Player.
\end{abstract}

Keywords: Web accessibility, video HTML 5, user agent, media players.

\section{Introduction}

The growth and access of rich and interactive contents such as video and audio have long filled the Web. Multimedia content must be accessible for people with disabilities according to standards like the Web Content Accessibility Guidelines (WCAG) [1] of Web Accessibility Initiative (WAI) [2]. Additionally, multimedia accessibility content on the Web requires that a particular chain of essential, interdependent, and accessible components [3] should be taken into account for user agents. Specifically, media players should enable the delivery of accessible multimedia content and therefore a friendly user-video interaction would be a success. Furthermore, it is imperative that Web designers are familiar with the User Agent Accessibility Guidelines (UAAG) [4] of WAI as well as the existing media players capable of the accessible delivery of multimedia content.

The UAAG explains how to make user agents - including Web browsers, media players, and assistive technologies - accessible for people with disabilities, and particularly, how to increase the accessibility of Web content. Related to multimedia contents, there are accessibility requirements that must be taken into account respect to audio and video on the Web. In general, media players must ensure that all audio

1 This research work is supported by the Research Network MAVIR (S2009/TIC-1542 (www.mavir.net/)), GEMMA (TSI-020302-2010-141) and SAGAS (TSI-020100-2010184) research projects. 
and video controls are accessible via keyboard and they should be accessed via screen readers. On the other hand, according the Guideline 1.2 of WCAG 2.0, media players must provide alternatives for time-based media and the media content must be accompanied by media alternatives as caption (or subtitles for deaf people), audio description, sign language, etc. in synchronized media. Moreover, according UAAG 2.0 guidelines, players should provide support for these media alternatives.

Previous studies [5] demonstrated that current media players are not sufficiently accessible. Most of the online videos are delivered via Adobe Flash ${ }^{2}$-based in-page video players. Flash has an excellent compression system that can deliver highfidelity audio and high-resolution video without taxing bandwidth. However, devices highly used for delivering video such as Apple ${ }^{3}$ - iPad and Apple - iPhone do not have support for this format.

As Universal alternative for solving accessibility problems appeared HTML5 [6]. This marked language allows video delivery on the Web to be independent from used user devices, introducing the <audio> and <video> elements, which enables web browsers to natively support media.

\section{Study of HTML 5 Support for Accessibility Standards}

The W3C is currently working on HTML 5, which will replace XHTML standards. HTML5 adds implicit semantic information, defines the basis of the documentoriented model (DOM), and presents a better structure. Regional landmarks on a webpage will have their own tags so that user agents can recognize them, which in turn will allow Web search robots gather more accurate information, letting authors embed accessibility multimedia elements natively (using <audio> and <video> tags), obviating the need for plug-ins.

HTML5 offers access in the embedded media player to be a huge step forward. The

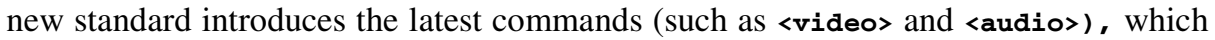
can create and label controls letting keyboard shortcuts to access them, and screen readers to tell the user which controls are available. Some HTML5 elements provide support for some UAAG 2.0 guidelines. However, the inclusion of caption and audio description, have not yet been included in the new standard because this is still under development. Problems have been detected in the current versions of most browsers in the support of <video> and <audio> labels ${ }^{4}$. Unfortunately, none of the current browser implementations are fully accessible by keyboard and screen-reader ${ }^{5}$. Others complications arise because the various browsers can't agree on what video codecs they're going to support, so actually using HTML5 <video> on the web is currently much more difficult than it should be ${ }^{6}$.

\footnotetext{
${ }^{2} 2011$ Adobe Systems Incorporated, http: / / www . adobe.com/

${ }^{3}$ Apple, http: / / www . apple.com/es /

${ }^{4}$ New HTML5 feature accessibility support in Windows Browsers,

http://html5accessibility.com/

${ }^{5}$ Keyboard and Screen-reader Accessibility

http://terrillthompson.blogspot.com/2010_08_01_archive.html

${ }^{6}$ Dive Into HTML5, What Works on the Web

http: / /diveintohtml5.org/video.html\#what-works
} 
The current version of HTML5 provides some playback controls for the video: play/pause, full screen toggle, volume and audio element controller toolbar. These controls are very basic, not allowing the user to have full control of the video. Following WCAG 2.0 and UAAG 2.0 guidelines, these native controls of HTML 5 are not enough. It must have the following controls: end (stop), caption on/off, search captions for text strings and select caption language if closed captioning is available, audio description on/off, rewind/forward seconds, volume up/ down, screen reader full access and keyboard full access of controls.

In this work, we have developed an accessible HTML5 Media Player ${ }^{7}$ as it is shown in the next section.

\section{Accessible HTML5 Media Player}

We have developed an accessible HTML5 Media Player, in order to build the mentioned controls as complement HTML5 support for interaction requirements included in UAAG 2.0, the use of JavaScript or Flash technology is required. We have chosen JavaScript because it does not require an external plug-in. Furthermore we have carried out different codifications of the same video to assure that the video can be played on all browsers. Currently there are three media formats that are under discussion of the different user agents: MP4, OGV, and WebM.

The attributes are included within <video> tag (see Figure 1), some of their values are called for JavaScript functions in order to achieve a high level of accessibility in the interaction with the video based on the standard UAAG 2.0. Some of the attributes are "controls" to load the native controls on devices with JavaScript disabled, and "onKeyDown" to control the video from the keyboard, so the user can use it for pause the video, stop it, show captions, etc. This function has been implemented with JavaScript.

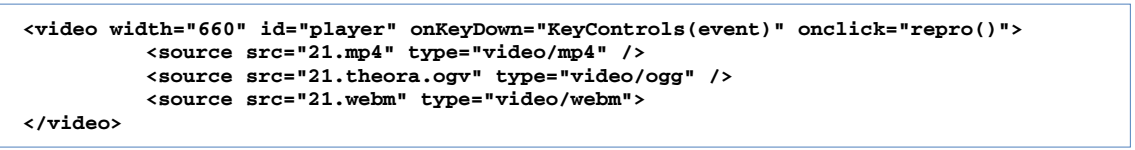

Fig. 1. HTML5 code of <video $>$

Controls have been incorporated through JavaScript, included in a separate $<$ div $>$ for the rest of the code, as it is shown in Figure 2.

<div id="controls" onMouseOut="HideControls () "onMouseOver="ShowControls () "

onkeyDown= "KeyControls (event) ">

Fig. 2. HTML5 code for controls

Because its complexity, it is not possible to describe in detail the code of each of the additional controls. Each of them has been implemented and its result is shown in Figure 3.

${ }^{7}$ Available in http: / / labda.inf .uc3m.es / LourdesPlayer / 
In order to add a caption in the player in HTML 5, an XML file was created with it. From this XML, file subtitles are being extracted and incorporated in a synchronized way through JavaScript and DOM is being modified.

In the case of access from devices with disabled JavaScript or CSS, the player will only appear with the HTML 5 native controls, allowing users access the video content. Moreover, to increase accessibility Keyboard Shortcuts have been defined to access the same functionality implemented using JavaScript technology and video transcriptions have also been provided. Finally, following UAAG requirements, which make reference to the usability, an alternative text have been associated for each of the player controls and the player offers a help guide, describing the keys associated with the keyboard shortcuts among others.

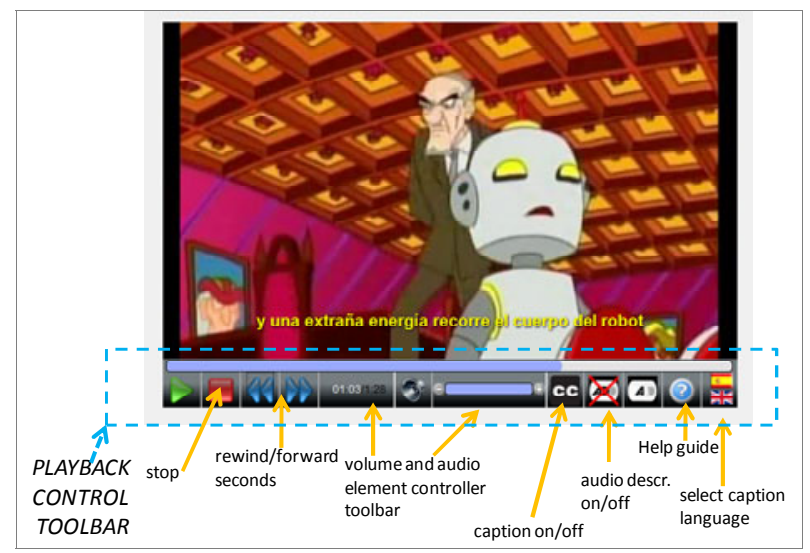

Fig. 3. Accessible HTML5 Media Player

\section{Conclusions}

HTML 5 represents a step forward in the multimedia history on the Web, but there is still a lot of work to do in order to include accessibility requirements on user interaction, such as those that were done explicitly in this work based on $\mathrm{W} 3 \mathrm{C}$ standard such as WCAG and UAAG. This issue is aggravated by the fragmentation between platforms. The question is posed, how should we deliver video, in Flash or HTML5? Currently, Flash supports more advanced interactions, but it presents some accessibility barriers. On the other hand, HTML5 provides accessible solutions, but it is still been developed and not all browsers implement it yet. Therefore, we suggest right now the smart strategy of implementing websites using both approaches, i.e.: approaches with accessible Flash players and with combination of HTML5 and JavaScript technology solution as it has been shown in this work. 


\section{References}

1. W3C, WAI, WCAG (2011), http: / /www .w3 .org/WAI / intro/wcag . php

2. W3C, Web Accessibility Initiative (WAI) (2011), http: / / www . w3 . org /WAI /

3. Moreno, L., Martínez, P., Ruiz-Mezcua, B.: Disability Standards for Multimedia on the Web. IEEE Multimedia 15(4) (2008)

4. W3C, WAI, UAAG (2011), http: / /www . w3 . org/WAI / intro/uaag • php

5. Moreno, L., Gonzalez, M., Martínez, P., Iglesias, A.: A Study of Accessibility Requirements for Media Players on the Web. In: Stephanidis, C. (ed.) HCII 2011 and UAHCI 2011, Part I. LNCS, vol. 6765, pp. 249-257. Springer, Heidelberg (2011)

6. W3C. HTML5. A vocabulary and associated APIs for HTML and XHTML. Working Draft (April 05, 2011), http://dev.w3 .org/html5/spec/Overview.html 